
About IJMA [last updated July, $\left.1^{\text {st }}, 2021\right]$

$\checkmark$ International Journal of Medical Arts is the Official Journal of the Damietta Faculty of Medicine, AlAzhar University, Egypt

$\checkmark$ It is an International, Open Access, Double-blind, Peer-reviewed Journal

$\checkmark$ Published four times a year

$\checkmark$ The First Issue was published in July 2019

$\checkmark$ Published under the following license: Creative Commons Attribution-ShareAlike 4.0 International Public License (CC BY-SA 4.0). It had updated from the Creative Commons license [CC BY] in volume 2, Issue 4, October 2020 About IJMA

$\checkmark$ The Egyptian Knowledge Bank hosts the web site of IJMA

$\checkmark$ The Egyptian Knowledge Bank supports IJMA

$\checkmark$ IJMA follows the regulations of the International Committee of Medical Journal Editors

$\checkmark$ IJMA is indexed in the "Directory of Open Access Journals" [15 January 2021].

$\checkmark$ IJMA is indexed in JGate [29-6-2021]

$\checkmark$ IJMA is a member of the International Society of Managing and Technical Editors

$\checkmark$ Listed in "Index Copernicus", "Publons", "Academic resource index [ResearchBib]", "Electronics journal library", "Eurasian Scientific Journal Index", and "Citefactor"

$\checkmark$ IJMA introduced to the search engine [BASE] through DOAJ
Click image to reach the page

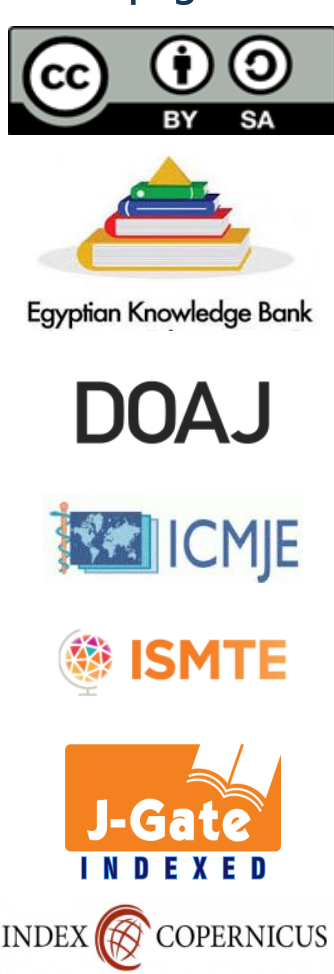

publons

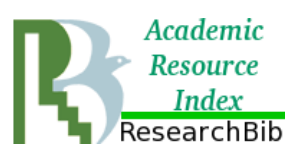

\section{EZ3 \\ .}

ESJII

CiteFactor



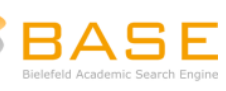




Available online at Journal Website
https://ijma.journals.ekb.eg/
Main subject [Neurosurgery]

Case Report

\title{
Intercostal Nerve Schwannoma as a Rare Cause of Refractory Mid Back Pain
}

\author{
Shahidul Islam Khan [1], Nazmin Ahmed [2], Mahamud Mannan [1], KM. Atiqul Islam [3], Vishal K. Chavda [4], \\ Gianluca Scalia [5], Santino Ottavio Tomasi [6], Giuseppe E. Umana [7], Bipin Chaurasia [8]
}

Consultant, Department of Orthopedic surgery, Bangabandhu Sheikh Mujib Medical University, Dhaka, Bangladesh [1]

Consultant, Neurosurgery, Ibn Sina Diagnostic and Consultation Center, Lalbag, Dhaka, Bangladesh [2]

Assistant Registrar, Department of Neurosurgery, National Institute of Neurosciences and Hospital, Dhaka, Bangladesh [3]

Division of Anesthesia, Sardar Women Hospital, Bapunagar, Ahmedabad, India [4]

Neurosurgery Unit, Highly Specialized Hospital and National Importance, Garibaldi, Catania, Italy [5]

Department of Neurological Surgery, Christian Doppler Medical Centre, Laboratory for Microsurgical Neuroanatomy, Paracelsus Medical University, Salzburg, Austria [6]

Department of Neurosurgery, Trauma and Gamma Knief Centre, Cannizzaro Hospital, Catania, Italy [7]

Consultant, Department of Neurosurgery, Bhawani Hospital and Research Centre, Birgunj, Nepal [8]

Corresponding author: Bipin Chaurasia

Email: trozexa@gmail.com

Submission date: April 05, 2021; Revision date: May 01, 2021; Acceptance date: May 03, 2021

Background: Schwannoma is the most common neurogenic tumor of the posterior mediastinum. Though commonly become asymptomatic, back pain with intercostal neuralgia mimicking post herpetic neuralgia is rare presentation and infrequently found in the available literature.

Case description: Here, we report a 22-year-old female without any history of trauma, surgery or infection presented with midback pain with radiation towards the right $10^{\text {th }}$ intercostal space. Although clinical evaluation simulates the features of post herpetic neuralgia, she was mildly responsive to traditional conservative treatment. Later on, magnetic resonance imaging [MRI] was performed to rule out the neoplastic cause which ultimately revealed Schwannoma of the right $10^{\text {th }}$ intercostal nerve. Patient symptomatically get well after complete removal of tumor. Beside this, we also briefly review the clinical presentation, neuroimaging features, different surgical options and outcome from the pertinent literature.

Conclusion: This report emphasizes early radiological evaluation of dull aching resting pain to avoid unnecessary medications to prolong duration.

Keywords: Schwannoma; Mid back pain; Post Herpetic Neuralgia; Peripheral nerves.

This is an open-access article registered under the Creative Commons, ShareAlike 4.0 International license [CC BY-SA 4.0] [https://creativecommons.org/licenses/by-sa/4.0/legalcode.

Citation: Khan SI, Ahmed N, Mannan M, Atiqul Islam KM, Chavda VK, Scalia G, Tomasi SO, Umana GE, Chaurasia B. Intercostal Nerve Schwannoma as a Rare Cause of Refractory Mid Back Pain. IJMA 2021; 3 [3] July-September: 1494-1499. [DOI: 10.21608/ijma.2021.71247.1294].

${ }^{*}$ Main subject and any subcategories have been classified according to the research topic. 


\section{INTRODUCTION}

A majority of posterior mediastinal masses are neurogenic in origin with schwannomas constituting $75 \%$ of benign nerve sheath tumors which is slow growing tumor that remains asymptomatic in most of the cases [1-3].

Without any extension into the spinal canal, refractory back pain and intercostal neuralgia including pain, tenderness, paresthesia, and hypoesthesia are one of the rarest presentations described in the available literature ${ }^{[4-5] \text {. }}$

Other presentation is persistent and progressive chest pain [5].

\section{Case description}

Here, we report a 22-year-old female without any history of trauma, surgery or infection presented with midback pain with radiation towards the right $10^{\text {th }}$ intercostal space.

Although clinical evaluation simulates the features of post herpetic neuralgia, she was mildly responsive to traditional conservative treatment. Later on, magnetic resonance imaging [MRI] was performed to rule out the neoplastic cause which ultimately revealed Schwannoma of the right $10^{\text {th }}$ intercostal nerve. Patient symptomatically get well after complete removal of tumor. Beside this, we also briefly review the clinical presentation, neuroimaging features, different surgical options and outcome from the pertinent literature.

History and physical examination: A 22 years old female from rural Bangladesh with no antecedent history of trauma, infection and surgery, presented with features of constant dull aching pain in the mid back region that referred towards the right $10^{\text {th }}$ intercostal space for one year. Clinically, she was diagnosed as a case of post-herpetic neuralgia, but she was refractory to several analgesics and anti-depressant medications. Beside this, the intensity of the pain hampered her dayto-day activities for six months. Therefore, she was evaluated radiologically and according to the findings, she was referred to our center for further evaluation and definitive management. After clinical evaluation, she was positive for Tinel sign during percussion of the $10^{\text {th }}$ intercostal space. Other neurological and systemic examination findings were normal.

Neuroimaging findings: Contrast-enhanced high resolution computed tomography [CT] scan of the chest showing a homogenous soft tissue mass in the right paravertebral space at the level of the $10^{\text {th }}$ intercostal space without calcification, having moderate contrast enhancement. No extension was is seen into the spinal canal [Figure: $1 A, B$ ]. On magnetic resonance imaging [MRI], the lesion was hypointense on $\mathrm{T} 1 \mathrm{WI}$, hyperintense on $\mathrm{T} 2 \mathrm{WI}$ with moderate heterogeneous contrast enhancement [Figure: $2 A, B]$.
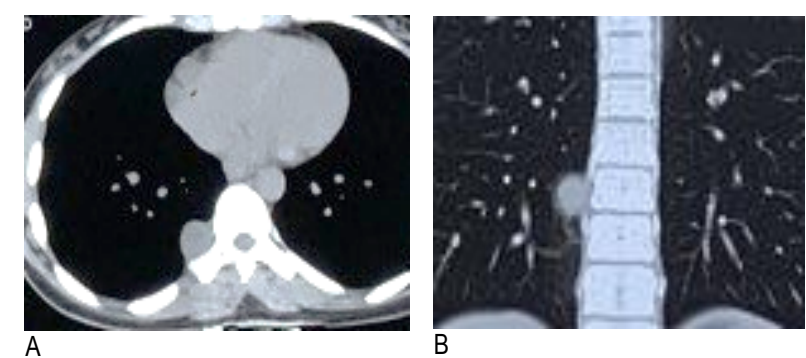

Figure [1]: Contrast enhanced high resolution CT scan of the chest showing a homogenous soft tissue mass in the right paravertebral space at the level of $10^{\text {th }}$ intercostal space without calcification, moderate contrast enhancement with no extension is seen into the spinal canal $[A, B]$

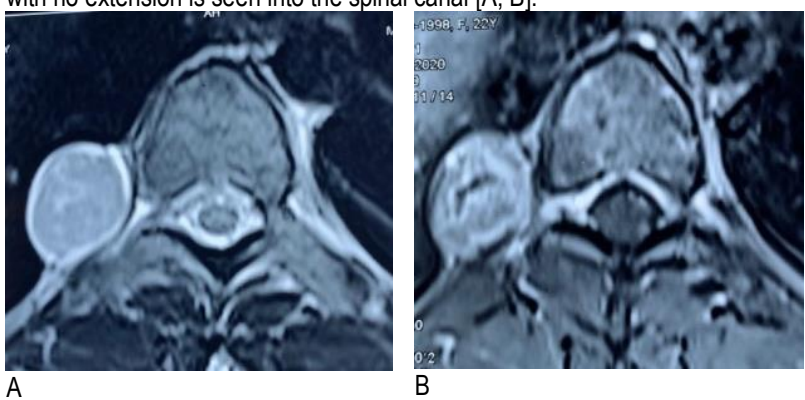

Figure 2: MRI of the dorsolumbar region with contrast demonstrates the lesion is hyperintense in T2WI with no marginal or intrinsic flow voids [A]; after injecting gadolinium there is heterogenous contrast enhancement with central necrotic area $[B]$.

Surgical procedure: An open posterior approach was used to expose the right side of the spine, from the spinous processes of D9 to D11 with right $10^{\text {th }}$ intercostal space done. A D10 hemilaminectomy with facetectomy and costotransversectomy was done. The proximal $4 \mathrm{~cm}$ of the tenth rib was resected and the partial shaving of the superior border of the eleventh rib was done to expose the tumor [Figure: $3 \mathrm{~A}, \mathrm{~B}$ ]. The entire tumor capsule was meticulously dissected from the surrounding structures. Tumor was completely excised after ligating the proximal and distal attachment to the intercostal nerve root. There was no per-operative injury to the pleura or dura. Considering unilateral approach into a single intercostal space and 
additional support by the rib cage, fixation and fusion was not done. Closure performed in layers maintaining the anatomical plane, leaving a subfascial drain in situ.
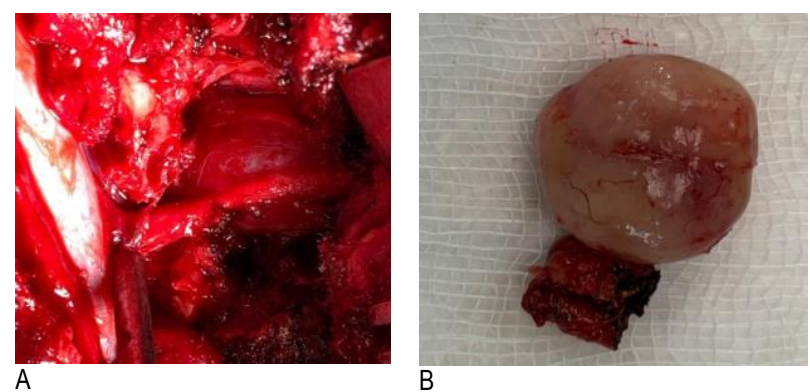

Figure 3: Per operative photograph showing the tumor attached with the intercostal nerve $[A]$; gross appearance demonstrates a whitish, smooth surface globular tumor $[\mathrm{B}]$

Postoperative period: The postoperative period was uneventful. Patient achieved complete relief from pain on $6^{\text {th }}$ post-operative day. There was no new neurological deficit observed. The biopsy report was consistent with Schwannoma [Figure: 4 and Figure: 5A, $\mathrm{B}, \mathrm{C}$, and D].

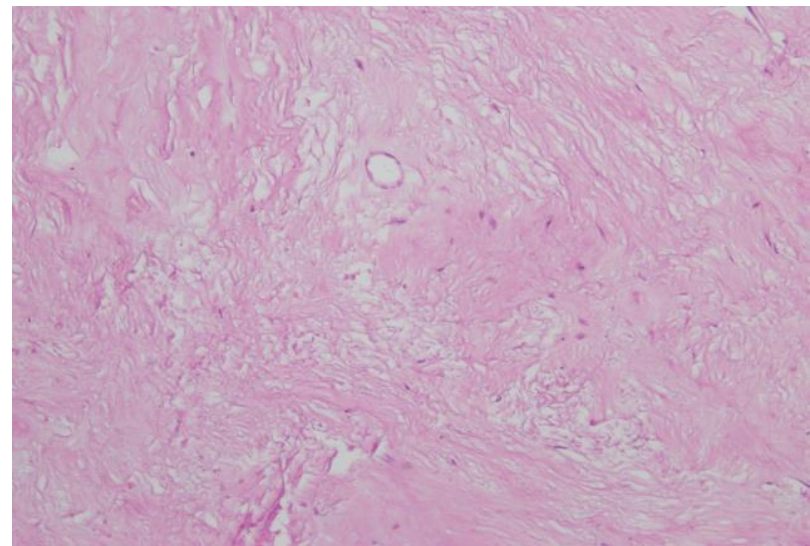

Figure [4]: Schwannoma staining with $\mathrm{H} \& \mathrm{E}[\mathrm{x} 400]$. The blue arrows point to a region with less cellularity compatible with a Verocay body. An area of Antoni A due to the presence of Verocay body and palisade area

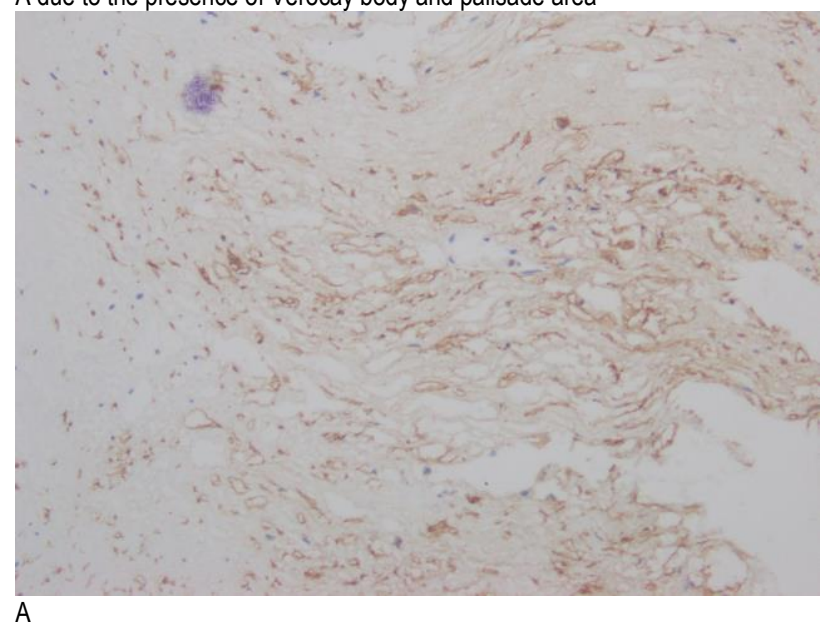

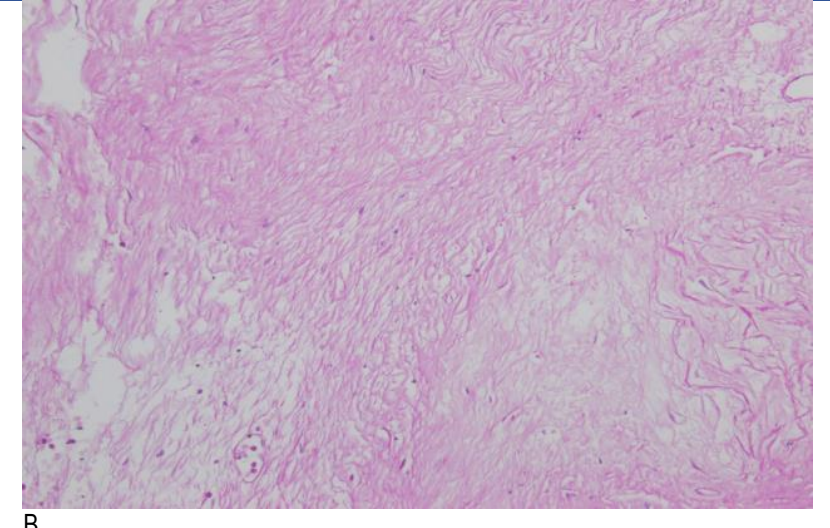

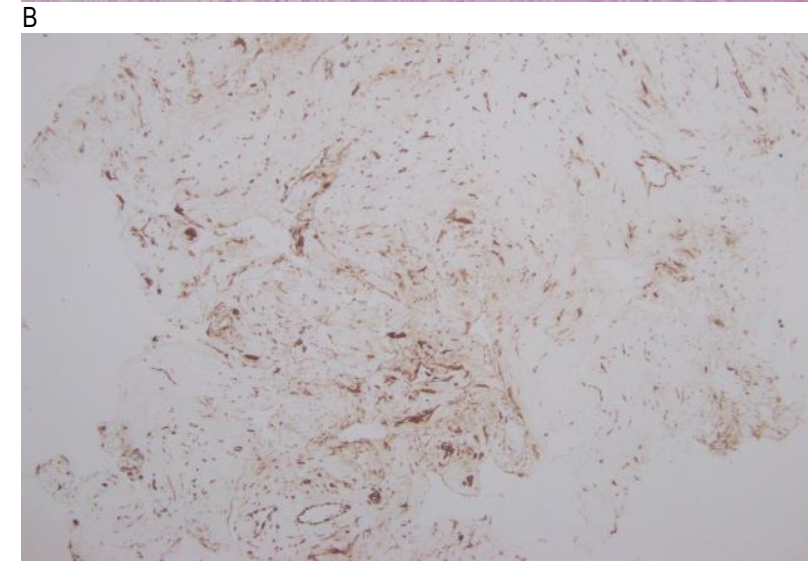

C

Figure [5]: Immunohistochemical analysis demonstrates positive staining for S$100[A]$ and PAS $[B]$, negative stain for vimentin [C] and Pan-CK [D].

\section{DISCUSSION}

Schwannomas arise from the Schwann cells of the neural sheath [3]. This tumor commonly located in the costovertebral sulcus in the paravertebral region in posterior mediastinum [6].

Although they presented as benign single lesion, atypical presentations, like-ancient schwannoma [6], cystic schwannoma [7-8], melanotic schwannoma [9], multiple schwannoma [10] following a single intercostal nerve and familial Schwannomatosis have been 
reported in the literature. Schwannomas are usually diagnosed in the $3^{\text {rd }}$ and $4^{\text {th }}$ decade of life with equal distribution to both sexes [3].

Schwannomas are often asymptomatic and incidentally detected during imaging $[6,11]$. However, after attaining a considerable size, patients may present with pain, tingling and numbness sensation along the distribution of the involved nerve [2,4]. Often these symptoms clinically mimic post-herpetic neuralgia and patients undergo long term treatment with different analgesics and antidepressant medications [4]. Beside this, the mass effect can be evident by compressing of adjacent mediastinal structures, such as lung, trachea, bronchi, heart, great vessels and patient may present with respiratory, cardiovascular of gastrointestinal symptoms [1,5,7,12-14].

This pattern of symptoms often biased the clinician about the definitive diagnosis. Very rarely, hemothorax [12], bloody pleural effusion [14], lung collapse ${ }^{[1]}$, massive pleural effusion ${ }^{[7]}$ and pericardial effusion [5] has been reported as an associated presentation.

Plain X-ray is one of the diagnostic tools for routine evaluation but mostly inconclusive if the tumor becomes insignificant. However, associated pleural and pericardial effusion, lung collapse can be diagnosed from X-ray which warrant the clinician for further evaluation of the etiology [15]. In case of dumbbell tumor with intraspinal extension, enlargement of the neural foramina, scalloping of posterior vertebral bodies can be seen [16]. More recently, ultrasonography [US] considered a fast and noninvasive approach to evaluate nerves which provide valuable information about the site and characteristics of peripheral nerve sheath tumor [4]. However, CT scan with intravenous contrast is the most valuable test for any mediastinal lesions to determine the location, relationship with surrounding structures. Beside this, it is the gold standard investigation to detect intralesional calcification and hemorrhage. Often this investigation can predict the lesion [3,15].

In our reported case, the lesion demonstrates well defined-margins, homogenous soft tissue mass, devoid of intralesional hemorrhage that goes mostly in favor of benign lesions. Though magnetic resonance imaging [MRI] is not routinely performed for mediastinal masses [15], it is advised in dumbbell lesions to see the extent of spinal canal extension as well as spinal cord compression [3]. Signal intensity varies due to the presence of cystic and hemorrhagic areas of the lesion $[5,17]$. Usually, schwannoma becomes iso to hypointense in $\mathrm{T} 1 \mathrm{Wl}$, hyperintense in $\mathrm{T} 2 \mathrm{Wl}$ with homogeneous contrast enhancement [6]. Rarely, this tumor becomes hyperintense in $\mathrm{T} 1 \mathrm{WI}$, due to the presence of melanin [9].

Heterogeneous contrast uptake is due to the presence of necrosis, cyst formation, calcification and intra-tumoral hemorrhage [1,5,9,15,17]. A definitive diagnosis is only possible after histopathological examination. Schwannoma and solitary fibrous tumor are both considered a differential diagnosis of spindle cell lesions [18].

Although they have different patterns, definitive diagnosis is challenging if not considering immunehistochemistry [14]. After staining with hematoxylin and eosin, areas of dense spindle cells [Antoni $A]$ and hypocellular stromal areas [Antoni B] became visible. Immunohistochemically, this tumor shows strong positivity to proteins, including S-100, vimentin and CD56, and negativity to desmin, smooth muscle myosin [SMA], CD34 and CD117. In both histological patterns of Schwannomas, the S-100 protein positivity is essential to establish the diagnosis. In our patient, the pathological specimen showed positivity to S-100 and PAS, negativity to vimentin and Pan-CK, hence definitive diagnosis was made.

Early surgical resection should be considered to avoid possibility of malignancy [19]. Moreover, surgery helps to stablish the definitive diagnosis. In case of small tumors without intraspinal extension, VATS is the procedure of choice [17,20-23]. Nowadays, VATS is regarded as a safe and reliable minimal access alternative to thoracotomy for managing posterior neurogenic tumor. This approach permits good exposure of the tumor, surrounding mediastinal structures, minimize tissue trauma, improved cosmetic appearance and a shorter hospital stay. Alternatively, thoracotomy can be considered if adequate facilities are not available, a large tumor [>6 cm], complex relationships with surrounding neuro-vascular 
structures, suspicious of malignancy and located at the costophrenic angle or thoracic apex. Before the surgical procedure, preoperative angiography helps identify the feeding arteries supplying both the tumor and spinal cord. Embolization helps in reducing tumor vascularity and operative blood loss which adds in safer resection [24]. Recently, Zhu et al. [25] introduced newer technique for the resection of lower posterior mediastinal tumors. To avoid the complications of VATS, they resected a benign posterior mediastinal schwannoma with retroperitoneo-scopy by transdiaphragmatic approach.

In case of large tumors with intraspinal extension, various approaches have been recommended including a single-stage posterior approach by laminectomy [26], hemilaminectomy with partial costotransversectomy [27-28], costotransversectomy with extension to a posterolateral thoracotomy [19] and combined posterior and transthoracic approach performed either in one or two stages [21]. Advantages of a single-stage posterolateral approach includes the necessity for single incision, known procedure to neurosurgeons, and avoiding a chest tube [19]. Though our reported tumor was $<6 \mathrm{~cm}$, located in the paravertebral space, possibility of its benign nature and no relationship with spinal cord, we went for hemi laminectomy with costotransversectomy due to lack of facilities for VATS and better familiarity of the surgical steps for a neurosurgeon. The necessity for instrumentation following single level costotransversectomy still a matter of debate [22]. However, considering the single level manipulation and additional support provided by the rib cage, we did not go for instrumentation. The complications such as bleeding, post-surgical infection or pleural injury are reported from $13 \%$ to $38 \%$ [27].

Though relatively higher chance of complications we had minimum perioperative hemorrhage with no pleural injury and post-operative infection. Untreated schwannomas usually continue to grow and become symptomatic by its mass effect on the nearby structures [3,17]. Rarely, they may show malignant transformation. Predictors of malignancy include rapid tumor growth and loss of neurologic function. In malignant schwannomas, radiotherapy may be required in addition to surgical treatment [3].
In our case, the lesion was surgically excised without any complications and the patient did not experience disease recurrence after 6 months of follow-up.

Conclusion: Intercostal nerve schwannoma should be considered as one of the differential diagnoses for intractable back pain and full diagnostic work up including neuroimaging is recommended because early surgical intervention is the only key for a favorable outcome.

\section{Financial and Non-Financial Relationships and Activities of Interest}

None

\section{REFERENCES}

1. Jang JY, Kim JS, Choe JW, Kim MK, Jung JW, Choi JC, Shin JW, Park IW, Choi BW, Kim JY. A case of giant, benign schwannoma associated with total lung collapse by bloody effusion. Tuberc Respir Dis [Seoul]. 2013 Aug;75[2]:71-4. [DOI: 10.4046/trd.2013.75.2.71].

2. Kermenli T, Ekiz T, Akarsu E, Çalık I, Yeğen SF. Persistent Back Pain Due to Intercostal Schwannoma. Pain Med. 2016 Apr:17[4]:791-3. [DOI: 10.1093/pm/ pnv028].

3. Magalhães MJ, Pereira DV, Oliva HN, Durães DT, Caiado FL, Araújo GM, Junior ET. Peripheral Nerve Schwannomas: A Literature Review. Arquivos Brasileiros de Neurocirurgia: Brazilian Neurosurgery. 2019; 38[04]:308-14. [DOI: 10.1055/ s-0039-1698786].

4. Coraci D, Nizegorodcew T, Aprile I, De Franco P, Padua L. Misdiagnosis of herpes zoster neuralgia: nerve ultrasound allowed diagnosing schwannoma of the intercostal nerve. Neurol Sci. 2014; 35[1]:131-3. [DOI: 10.1007/s10072-0131517-3]

5. Chen X, Ma Q, Wang S, Zhang H, Huang D. Surgical treatment of posterior mediastinal neurogenic tumors. J Surg Oncol. 2019 May;119[6]:807-813. [DOI: 10.1002/jso.25381].

6. Shanmugasundaram $G$, Thangavel $P$, Venkataraman $B$, Barathi G. Incidental ancient schwannoma of the posterior mediastinum in a young male: a rare scenario. BMJ Case Reports CP. 2019 May 1;12[5]: e227497. [DOI: 10.1136/bcr2018-227497].

7. Mubashir M, Salam A, Sonawalla A, Fatima H, Fatimi SH. Rare presentation of a posterior mediastinal cystic schwannoma as a large pleural effusion. Cureus. 2017; 9: e1558. [DOI: 10.7759/cureus.1558].

8. Sayit AT, Elmali M. Rare presentation of a posterior mediastinal cystic schwannoma. Cukurova Medical Journal.,45[2]:760-2. [DOI: 10.17826/cumj.700358]. 
9. Hijiya K, Sakamoto J, Kosaka S, Nagasako R. Psammomatous Melanotic Schwannoma Not Associated with Carney Complex: A Case Report. Japanese Journal of Lung Cancer. 2019; 59: 244-247

10. Chen F, Nakayama E, Okubo K, Date H. Intrathoracic multiple schwannomas of a single intercostal nerve. The Annals of thoracic surgery. 2008; 86 [2]: 660-1. [DOI: 10.1016/j. athoracsur.2008.02.004]

11. Watkins $R$ 4th, Watkins R 3rd, Williams L, Ahlbrand S, Garcia R, Karamanian A, Sharp L, Vo C, Hedman T. Stability provided by the sternum and rib cage in the thoracic spine. Spine [Phila Pa 1976]. 2005 Jun 1;30 [11]: 1283-6. [DOI: 10.1097/01.brs.0000164257. 69354.bb].

12. Amin R, Waibel BH. An unusual presentation of a posterior mediastinal schwannoma associated with traumatic hemothorax. Case Rep Surg. 2015; 2015: 175645. [DOI: 10.1155/2015/175645].

13. Kwak YJ, Park S, Kang CH, Kim YT, Park IK. Development of Castleman Disease in the Paravertebral Space Mimicking a Neurogenic Tumor. Korean J Thorac Cardiovasc Surg. 2019 Feb;52[1]:51-54. [DOI: 10.5090/kjtcs.2019.52.1.51].

14. Nosrati R, Anissian D, Ramezani F, Sharbatdaran M. Benign schwannoma of posterior mediastinum accompanied by bloody pleural effusion misdiagnosed as solitary fibrous tumor: A case report. Caspian J Intern Med. Fall 2019;10[4]:468-471. [DOI: 10.22088/ cjim.10.4.468].

15. Loftus TJ, Pipkin M, Machuca T, Oduntan O. Angiographic embolization followed by piecemeal resection of giant posterior mediastinal schwannoma: Case report and concise review. Int J Surg Case Rep. 2018; 53:250-3. [DOI: 10.1016/j.jisscr.2018.10.055].

16. Ando K, Imagama S, Ito Z, Tauchi R, Muramoto A, Matsui $H$, Matsumoto $\mathrm{T}$, Ishiguro $\mathrm{N}$. Removal of thoracic dumbbell tumors through a single-stage posterior approach: its usefulness and limitations. J Orthop Sci. 2013 May; 18 [3]: 380-7. [DOI: 10.1007/ s00776-013-0370-9].

17. Khanlou H, Khanlou N, Eiger G. Schwannoma of posterior mediastinum: a case report and concise review. Heart Lung J Crit Care. 1998; 27:344-7. [DOI: 10.1016/s0147-9563 [98]90055-3].

18. de Barros LM, de Oliveira AJM, Santos AS, Lima FL, Valente RS. Peripheral nerve entrapments-rare causes of a common condition: case series. Autops Case Rep. 2020 May 6;10[2]: e2020153. [DOI: 10.4322/acr.2020. 153].

19. Moses ZB, Barzilai O, O'Toole JE. Benign Intradural and Paraspinal Nerve Sheath Tumors: Advanced Surgical Techniques. Neurosurg Clin N Am. 2020 Apr;31[2]:221-229. [DOI: 10.1016/j.nec.2019.11.002].
20. Liu HP, Yim AP, Wan J, Chen H, Wu YC, Liu YH, Lin PJ, Chang $\mathrm{CH}$. Thoracoscopic removal of intrathoracic neurogenic tumors: a combined Chinese experience. Ann Surg. 2000 Aug;232[2]:187-90. [DOI: 10.1097/ 00000658200008000-00006].

21. Nam KH, Ahn HY, Cho JS, Kim YD, Choi BK, Han IH. One Stage Posterior Minimal Laminectomy and Video-Assisted Thoracoscopic Surgery [VATS] for Removal of Thoracic Dumbbell Tumor. J Korean Neurosurg Soc. 2017 Mar;60[2]:257-261. [DOI: 10.3340/jkns.2016. 0909.004].

22. Takeda S, Miyoshi S, Minami M, Matsuda H. Intrathoracic neurogenic tumors--50 years' experience in a Japanese institution. Eur J Cardiothorac Surg. 2004 Oct;26[4]:807-12. [DOI: 10.1016/j.ejcts.2004. 07.014].

23. Yamaguchi M, Yoshino I, Fukuyama S, Osoegawa A, Kameyama T, Tagawa T, Maehara Y. Surgical treatment of neurogenic tumors of the chest. Ann Thorac Cardiovasc Surg. 2004 Jun;10[3]:148-51. [PMID: 15312009].

24. Loftus TJ, Pipkin M, Machuca T, Oduntan O. Angio-graphic embolization followed by piecemeal resection of giant posterior mediastinal schwannoma: Case report and concise review. Int J Surg Case Rep. 2018; 53:250-253. [DOI: 10.1016/j.j.jscr.2018.10.055].

25. Zhu D, Hong P, Chen S, Fan Y, Zhang Z, Zhang X, Yu W, Shen $C$, Jin J. Lower posterior mediastinal benign schwannoma successfully resected with retroperitoneoscopy using a transdiaphragmatic approach: A case report. Thorac Cancer. 2018 Aug; 9 [8]:1087-1089. [DOI: 10.1111/1759-7714.12786].

26. Chen X, Ma Q, Wang S, Zhang H, Huang D. Surgical treatment of posterior mediastinal neurogenic tumors. J Surg Oncol. 2019 May;119[6]:807-813. [DOI: 10.1002/jso.25381].

27. Marhx Bracho A, Muñoz Montoya JE, Peña Rosas NP, Carrillo Marhx G, Ramírez Ferrer E. Costotransverse-ctomy plus hemilaminectomy as alternative surgical approach for extramedullary intradural thoracic schwannoma resection with and without extradural extension in pediatric population three cases and literature review. J Spine Surg. 2019 Jun; 5[2]:285-290. [DOI: 10.21037/jss.2019.05.07].

28. Takamura $Y$, Uede $T$, Igarashi $K$, Tatewaki $K$, Morimoto $S$. Thoracic dumbbell-shaped neurinoma treated by unilateral hemilaminectomy with partial costo-transverseectomy--case report. Neurol Med Chir [Tokyo]. 1997 Apr;37[4]:354-7. [DOI: 10.2176/nmc. 37.354]. 

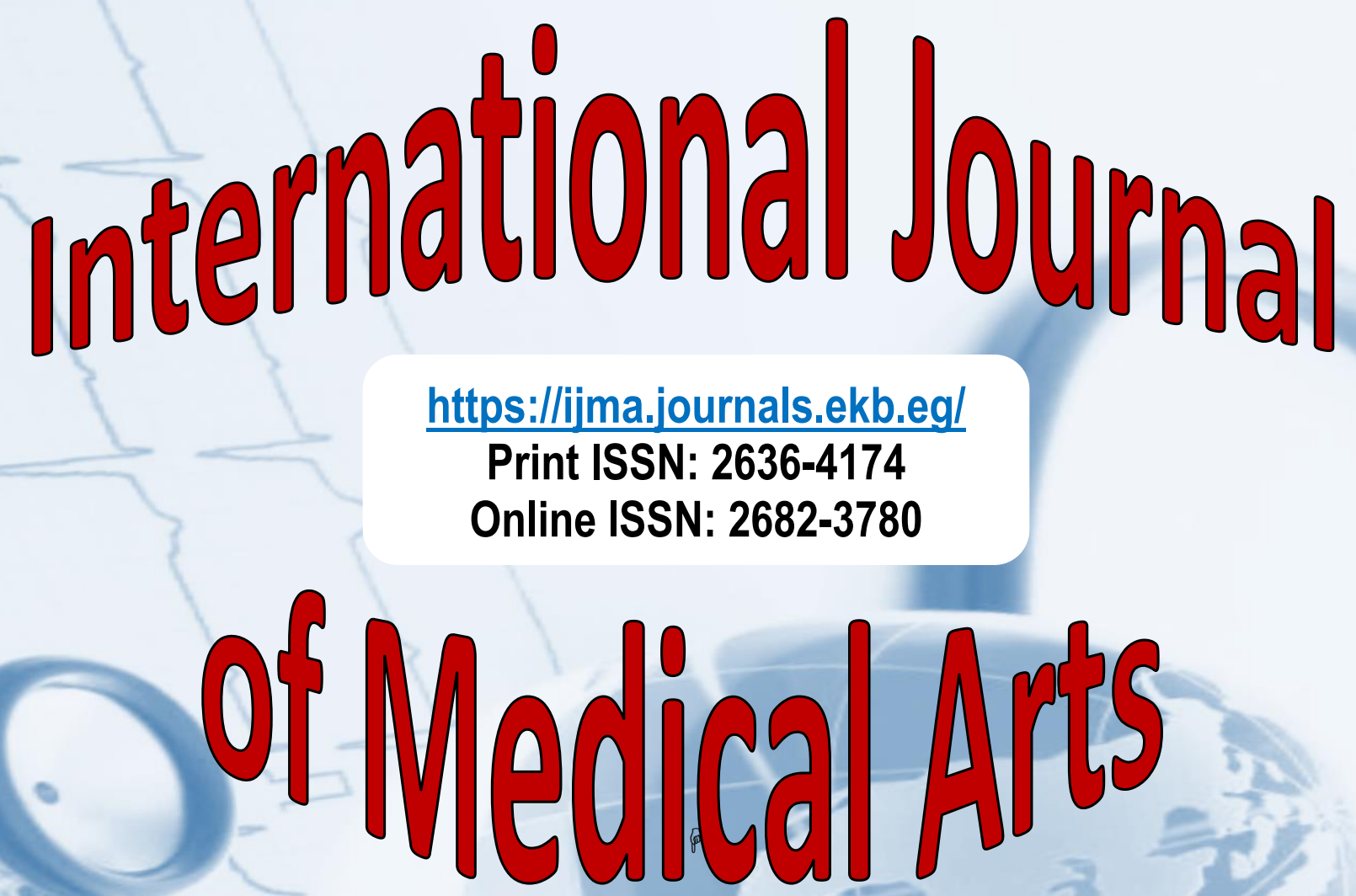